\title{
Thermal Transport of Flexural and In-Plane Phonons Modulated by Bended Graphene Nanoribbons
}

\author{
Changning Pan, ${ }^{1,2}$ Jun He, ${ }^{1}$ Diwu Yang, ${ }^{1}$ and Keqiu Chen ${ }^{3}$ \\ ${ }^{1}$ School of Science, Hunan University of Technology, Zhuzhou 412008, China \\ ${ }^{2}$ Hunan Province Higher Education Key Laboratory of Modeling and Monitoring on the Near-Earth Electromagnetic Environments, \\ University of Science and Technology, Changsha 410004, China \\ ${ }^{3}$ Department of Applied Physics, Hunan University, Changsha 410082, China
}

Correspondence should be addressed to Changning Pan; panchangning2000@sina.com and Jun He; hejun@hnu.edu.cn

Received 26 December 2015; Accepted 7 April 2016

Academic Editor: Yasuhiko Hayashi

Copyright (c) 2016 Changning Pan et al. This is an open access article distributed under the Creative Commons Attribution License, which permits unrestricted use, distribution, and reproduction in any medium, provided the original work is properly cited.

Ballistic thermal transport properties are investigated comparatively for out-of-plane phonon modes (FPMs) and in-plane phonon modes (IPMs) in bended graphene nanoribbons (GNRs). Results show that the phonon modes transports can be modulated separately by the phonon dispersion mismatch between armchair and zigzag GNRs in considered system. The contribution of FPMs to total thermal conductance is larger than $50 \%$ in low temperature for perfect GNRs. But it becomes less than $20 \%$ in the bended GNRs. Furthermore, this contribution can be modulated by changing the structural parameters of the bended GNRs. The result is useful for the design of thermal or thermoelectric nanodevices in future.

\section{Introduction}

Graphene, a monolayer of carbon atoms honeycomb lattice, has attracted much attention due to its unique electronic and thermal properties. It has been recognized as one of the most promising candidates for next generation electronics [1-7]. The measured thermal conductivity ranges from 600 to $5000 \mathrm{~W} / \mathrm{mK}$ [8-11]; it is almost an order of magnitude higher than that of the silicon counterpart and well consistent with theoretical calculation [12, 13]. In recent years, graphene-based thermal devices have been intensively investigated in theoretical and experimental aspects. For instance, negative differential thermal conductance and nonlinear thermal transport of graphene nanoribbons (GNRs) have been explored [14]; tunable thermal conductance has been observed in folded graphene and extended defects $[15,16]$; the effective thermal rectifications have been found in asymmetric three-terminal graphene nanojunctions and asymmetric GNRs [17-19]. In addition, enhanced thermoelectric properties have also been predicted in the mixed edges GNRs [20], edge disorder GNRs [21], kinked GNRs [22, 23], stub GNRs
[24], antidot lattices $[25,26]$, and graphene-nanoribbonbased heterojunctions [27]. Meanwhile, the previous studies also show that thermal transport properties of graphene always exhibit sensitive structure-dependent such as defects [28-30] and cavities [31]. All of the above investigations, the total thermal properties, have been studied. As is wellknown, lattice vibrations in the graphene can be classified as the in-plane phonon modes (IPMs) which vibrate in the plane of the layer with linear transverse and longitudinal acoustic branches and the out-of-plane phonon modes or the so-called flexural phonon modes (FPMs), which vibrate out of the plane of the layer [32]. The two types of phonon modes are decomposing in the thermal transport. One can calculate separately contributions of each type of mode to the total thermal conductance. Based on the exact numerical solution of the phonon Boltzmann equation, the authors studied the lattice thermal conductivity of graphene and found that the contribution is dominated by FPMs [32]. Recently, the researchers also found that the FPMs displayed the significant contribution to the total thermal conductivity in grain boundary GNRs [33]. The results in simulation are in 


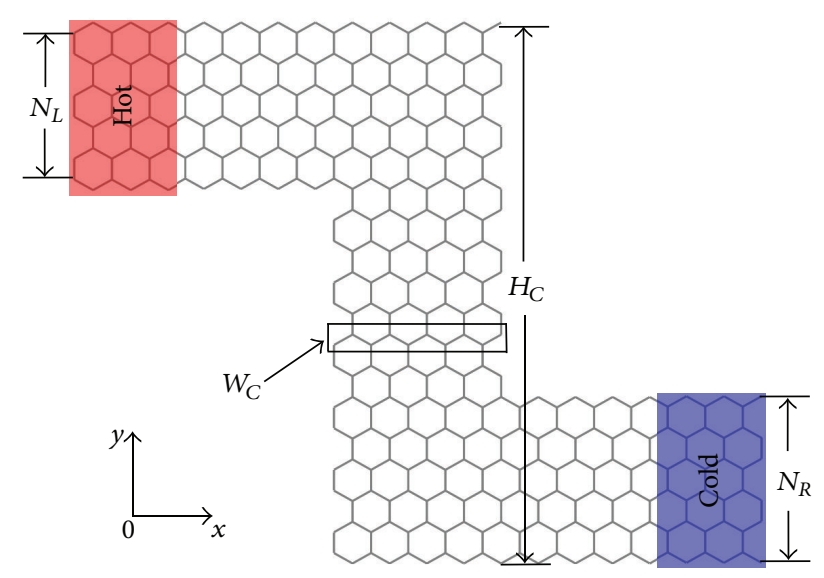

FIGURE 1: Schematic diagram of the bended graphene nanoribbons.

agreement with that in measurement of thermal conductivity on supported graphene [34].

However, most of the previous researches thus far investigate the total thermal conductance of the graphene and graphene-based devices or only focused on the thermal transport properties of FPMs for the graphene and GNRs. The systematic investigation of two types of phonon modes transport and how to tune separately their transport properties by thermal devices are paid less attention. In the present work, we study the modulated phonon transport and thermal conductance of IPMs and FPMs in bended GNRs by using the NEGF approach [35]. The results show that the quantum plateau of the reduced thermal conductance, $\pi^{2} k_{B}^{2} T / 3 h$ for FPMs, is easy to be destroyed by the nonmatching phonon modes in bended structure. The contribution of FPMs to the total thermal conductance declined sharply in low temperature and is dependent on the structural parameters. The thermal transport of FPMs is weaker than that of IPMs in the lowenergy quantum transport, but it becomes more robust in the high-energy classical transport.

\section{Model and Formalism}

Figure 1 shows the schematics of the considered geometry in the $x-y$ plane. The system is divided into three regions: the left/right (L/R) semi-infinite leads which are connected to two reservoirs and the central scattering region $(\mathrm{C})$, that is, bended GNR section. The width of the lead is $N_{L / R}$, the interbend length is $H_{C}$, and width is $W_{C}$. The phonon-phonon interaction has been neglected due to the large phonon meanfree path of graphene [36]. Using Brenner's empirical potential [37], the force constants between the carbon atoms in different direction can be obtained.

In harmonic approximation, the Hamiltonian for both IPMs and FPMs is completely decoupling and can be described separately as the sum of kinetic energy and potential energy of phonon [38]:

$$
H_{\beta}=\sum_{\alpha=L, C, R} H_{\alpha, \beta}+\left(u_{\beta}^{L}\right)^{T} V_{\beta}^{L C} u_{\beta}^{C}+\left(u_{\beta}^{C}\right)^{T} V_{\beta}^{C R} u_{\beta}^{R},
$$

where $u_{\beta}^{\alpha}$ is a column vector consisting of the reduced vibrational displacement of each atom in $\alpha$ region of $\beta$ vibration ( $\beta$ is $x, y$, or $z$ ). $V_{\beta}^{L C}$ and $V_{\beta}^{C R}$ are the spring constant matrix for the coupling between center and left/right leads. $H_{\alpha, \beta}$ represents the harmonic oscillators Hamiltonian for region $\alpha$ in $\beta$ direction:

$$
H_{\alpha, \beta}=\frac{1}{2}\left(\dot{u}_{\beta}^{\alpha}\right)^{T}+\frac{1}{2}\left(u_{\beta}^{\alpha}\right)^{T} K_{\alpha, \beta} u_{\beta}^{\alpha},
$$

where $K_{\alpha, \beta}$ is the force constant matrix in $\alpha$ region of $\beta$ vibration. Retarded Green's function for the center region connected with leads:

$$
\mathbf{G}_{\beta}^{r}=\left[(\omega+i \eta)^{2} I-K_{C, \beta}-\Sigma_{L, \beta}^{r}-\Sigma_{R, \beta}^{r}\right]^{-1} .
$$

The retarded self-energy $\boldsymbol{\Sigma}_{L(R), \beta}^{r}$, which carries the coupling information between center region and leads, can be expressed as $\Sigma_{L(R), \beta}^{r}=V_{\beta}^{C, L(R)} g_{L(R), \beta}^{r} V_{\beta}^{L(R), C}$, where $g_{L(R), \beta}^{r}$ are retarded surface Green's function of IPMs and FPMs for the two (left/right) isolated leads and can be calculated as

$$
g_{L(R), \beta}^{r}=\left[(\omega+i \eta)^{2} I-K_{L(R), \beta}\right]^{-1},
$$

where $\eta \rightarrow 0$ is a constant and $I$ is a unit matrix.

The phonon transmission function and phonon density of states (PDOS) on the $i_{\text {th }}$ atom in the central region can be derived for IPMs and FPMs:

$$
\begin{aligned}
T_{\beta}(\omega) & =\operatorname{Tr}\left(\mathbf{G}_{\beta}^{r} \boldsymbol{\Gamma}_{L, \beta}^{r} \mathbf{G}_{\beta}^{a} \boldsymbol{\Gamma}_{R, \beta}^{r}\right), \\
\rho_{i, \beta}(\omega) & =-\frac{2 \omega}{\pi} \operatorname{Trace}\left(\operatorname{Im}\left[\mathbf{G}_{\beta}^{r}\right]_{i i}\right),
\end{aligned}
$$

where advanced Green's function $\mathbf{G}_{\beta}^{a}=\left(\mathbf{G}_{\beta}^{r}\right)^{\dagger}$. The $\boldsymbol{\Gamma}$ function is $\boldsymbol{\Gamma}_{L(R), \beta}^{r}=-2 \operatorname{Im} \boldsymbol{\Sigma}_{L(R), \beta}^{r}$. After obtaining the transmission function of the systems, the corresponding thermal conductance contributed from the IPMs or the FPMs can be derived as

$$
\kappa_{\beta}=\frac{\hbar^{2}}{2 \pi k_{B} T^{2}} \int_{0}^{\infty} \omega^{2} \frac{e^{\hbar \omega / k_{B} T}}{\left(e^{\hbar \omega / k_{B} T}-1\right)^{2}} T_{\beta}(\omega) d \omega,
$$

with $k_{B}$ being the Boltzmann constant.

\section{Numerical Results and Discussion}

In Figure 2, we display the frequency-dependent phonon transmission coefficients of IPMs and FPMs for the perfect ZGNRs with width $N_{z}=6$ (6-ZGNR) and bended graphene with $N_{L / R}=6, H_{c}=18$, and $W_{c}=5$, respectively. In perfect 6-ZGNR, the transmission curves for IPMs and FPMs appear to be the smooth stepwise platforms. It is shown that the higher frequency phonon modes are excited and the more phonon passages are available in the high temperature. But the cut-off frequency of two kinds of the phonon modes have obviously different characteristics. The foremost lowest frequencies FPMs always have lower cut-off frequencies than those of the IPMs. Particularly for the 0th mode, the cut-off 


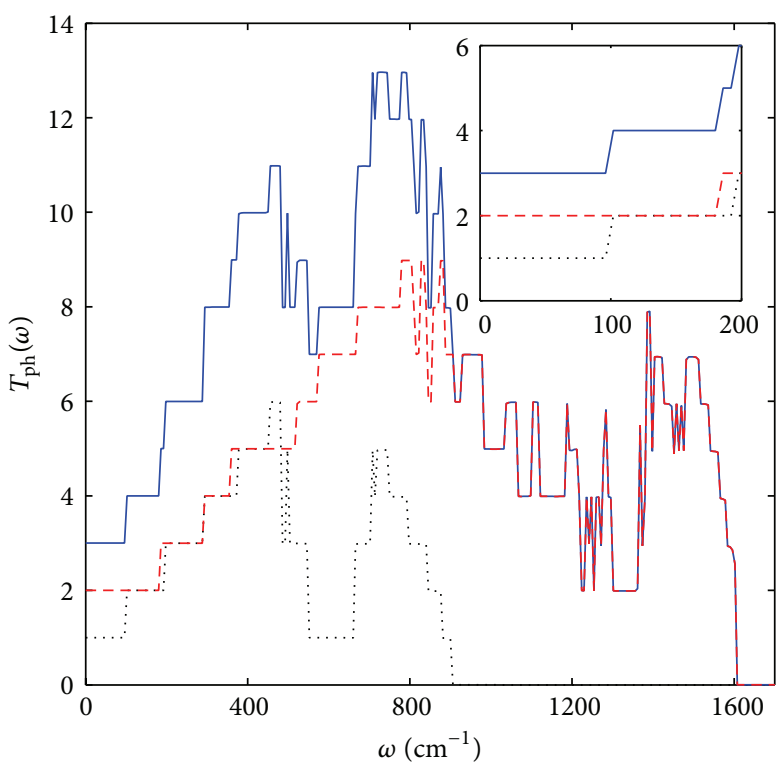

(a)

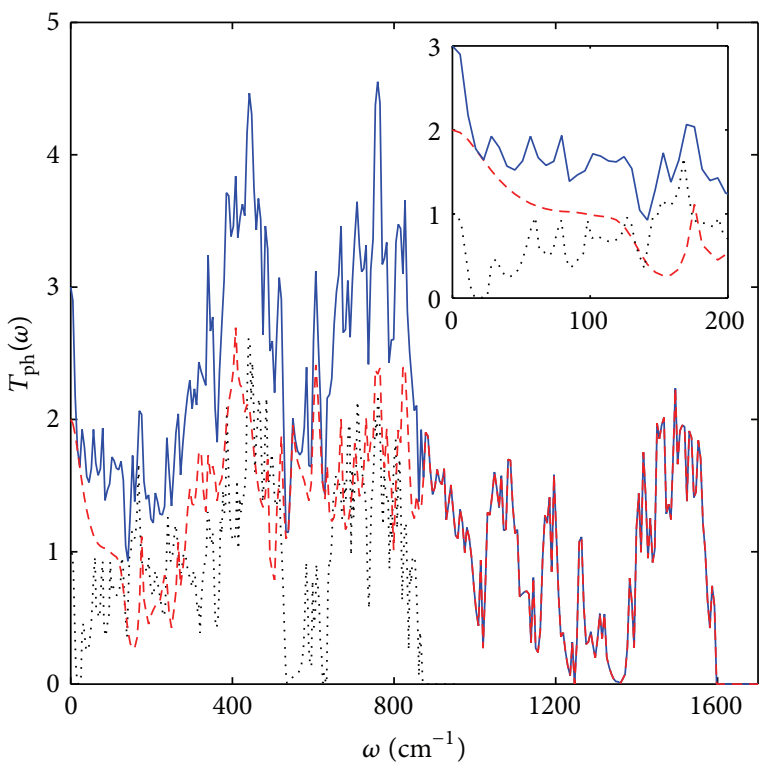

(b)

FIGURE 2: Phonon transmission functions for (a) the perfect 6-ZGNR and (b) bended GNR with leads $N_{L}=N_{R}=18$, the interbend length $H_{c}=6$, and width $W_{c}=5$. Solid, dashed, and dotted curves correspond to the total, IPMs, and FPMs transmission rate, respectively.

frequency is about $180 \mathrm{~cm}^{-1}$ for IPMs, which is twice wider than the FPMs about $96 \mathrm{~cm}^{-1}$. The phonon energy spectrum scope of IPMs is wider and ranges from zero to $1608 \mathrm{~cm}^{-1}$, while it is only $906 \mathrm{~cm}^{-1}$ for FPMs. For the bended GNRs, though the phonon transmission coefficient $T_{\mathrm{ph}}(\omega)$ in the low temperature limit takes the quantum value 2 for IPMs and 1 for FPMs as the perfect GNRs, we find that the stepwise platforms of two kinds of phonon transport are dramatically destroyed by the bended structure. Many deep transmission dips arise at some frequencies, which are clearly different from the dips originating from the van Hove singularity of the optical phonon modes. In particular, the geometry effect is much stronger for the FPMs. For example, the transmission rate $T_{\mathrm{ph}}(\omega) \rightarrow 0$ at $\omega=22 \mathrm{~cm}^{-1}$, this means that the phonon passages have been prohibited at this frequency, and the incident phonons have been fully scattered by the structure. Such strong scatterings result in the fact that the transmission of FPMs is obviously lower than that of IPMs.

To further interpret the physical mechanism in which the scattering of FPMs is stronger than that of IPMs, the phonon density of states (PDOS), which can directly show the phonon transport information of each atom, have been calculated for both the FPM and IPM at $\omega=22 \mathrm{~cm}^{-1}$, as shown in Figure 3. From Figure 3(a), we can clearly see that some PDOS of FPMs are highly localized at both ends of the system and form the phonon localized states. The interbend section (the armchair edge GNR) has no distributions of PDOS. The local distributions of phonons demolish effectively the transmission of FPMs and lead to the low transmission rate. Indeed, the considered system is composed by zigzag- or armchairedged GNRs linked alternatively. As is known, these two types of perfect GNRs have different phonon dispersion relations [39]. It gives rise to the mismatches of modes in phonon transport. The strong phonon scattering occurs in some frequency region. Therefore, the armchair GNR sandwiched between two zigzag GNRs can be seen as a barrier and acts as the phonon scatterer in considered system. It holds back a certain of frequency incoming phonon of FPMs and leads to $T_{\mathrm{ph}}(\omega) \rightarrow 0$. However, the PDOS of the IPM are delocalized over the whole system as in Figure 3(b). The wellproportioned distributions of IPMs result in the fact that the incoming phonons can pass through the system with less scattering. Similar behaviors can also be observed in the pentagon-heptagon defect [38].

Next, we turn to study specially the thermal conductance of the system, in order to compare the thermal conductance of FPMs with that of IPMs. The temperature-dependent thermal conductance of each kind of phonon mode was plotted in Figure 4, separately. We find that all types of the thermal conductance increase monotonically with rising temperature in both the perfect 6-GNR and the bended GNR. It is because the high-energy phonons are excited in the high temperature. However, the value of thermal conductance for FPMs and IPMs appears to be different behaviors. For the 6-GNR, the thermal conductance of the FPMs is very close to that of IPMs in the low temperature region $T<138 \mathrm{~K}$ and then becomes less than that of IPMs in the high temperature. It is because the FPMs with the narrow cut-off frequency can be excited easily and contribute to the thermal conductance. But in the high temperature, owing to the wide energy spectrum of IPMs, the more IPMs are excited in the high frequency region, which result in the higher thermal conductance. However, the thermal conductance of FPMs is always lower than that of IPMs for the bended GNR. The major reason, which is unlike the electron transport, is the low-energy phonons which contribute most to the thermal transport. But, as 


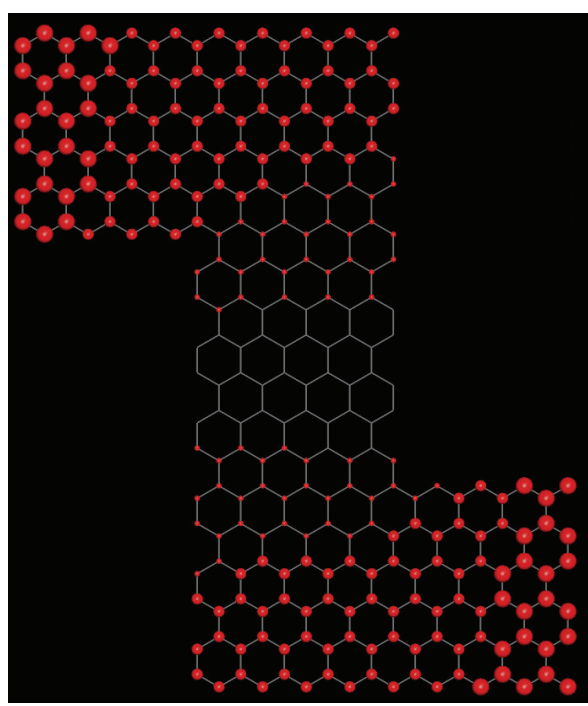

(a)

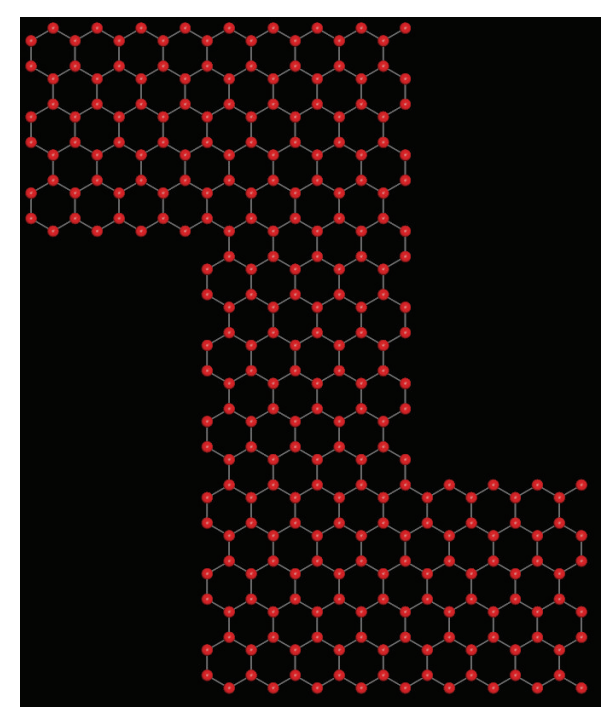

(b)

FIgURE 3: The PDOS of (a) the FPMs and (b) IPMs at $\omega=22 \mathrm{~cm}^{-1}$ for the bended GNRs $N_{L}=N_{R}=6, H_{c}=18$, and $W_{c}=5$.

mentioned above, the low frequency phonons of FPMs are dramatically scattered by the mismatch of phonon modes. It yields low thermal conductance. For instance, the thermal conductance is $0.02 \mathrm{nW} / \mathrm{K}$ for FPMs in the temperature $T=$ $30 \mathrm{~K}$; it is about three times less than the thermal conductance of IPMs with $0.07 \mathrm{nW} / \mathrm{K}$. In the inset of Figure 4, we calculate the reduced thermal conductance as a function of the temperature dependence. It can be found that the reduced thermal conductance approaches the quantum value $1\left(\pi^{2} k_{B}^{2} T / 3 h\right)$ for FPMs and the quantum value $2\left(\pi^{2} k_{B}^{2} T / 3 h\right)$ for IPMs in the low temperature limit, regardless of the perfect $6-\mathrm{GNR}$ or the bended GNRs. In fact, it can be well understood from the formula $T=\hbar \omega / k_{B}=h c / K_{B} \lambda$ [40]. The wavelength of phonons increases with the decrease of the temperature. When the temperature $T \rightarrow 0$, only the 0th acoustic mode is excited; the wavelength of the phonons becomes much larger than the dimension of the central scattering region of the bended GNR. As a result, the long-wavelength phonons can pass through perfectly in the bended GNR and exhibit quantum thermal value. Meanwhile, we find that, due to the more number of excited modes, the reduced thermal conductance of FPMs is increasing rapidly with the temperature and is larger than that of IPMs in the temperature ranged from the $40 \mathrm{~K}$ to $100 \mathrm{~K}$ for the perfect 6-GNR. Nevertheless, as shown in the inset of Figure 4(b), the quantum plateau of FPMs and IPMs has been significantly destroyed by the scattering of bended GNR. The reduced thermal conductance degraded with the increasing temperature. In particular, the distinct dip occurs at the temperature $T=60 \mathrm{~K}$ in reduced thermal conductance curve of FPMs.

Since FPMs is very important for the thermal transport in GNRs, to check how much contribution it is, we calculate the temperature dependence contribution of these modes to total thermal conductance. Figure 5 shows the contribution of FPMs for different interbend length $H_{c}$. In the low temperature limit, $T \rightarrow 0$, the thermal contributions always equal $1 / 3$, regardless of perfect $6-$ GNR and bended GNR. This is because three lowest acoustic modes, that is, two coupling lowest transversal acoustic and longitudinal acoustic modes and the lowest FPM, can be transported smoothly. But the contribution of FPMs in the perfect 6-ZGNR increases rapidly with the increasing temperature and then decreases dramatically. The peaks value is more than $50 \%$ in low temperature and then less than $30 \%$ in high temperature. In contrast, for the bended structure, this contribution decreases dramatically in low temperature due to the intense scattering which originates from the mode mismatch, then increases slowly, and finally becomes nearly independent of the temperature. The valley value with almost $20 \%$ appears at temperature $T=60 \mathrm{k}$ and the contribution of FPMs is above $40 \%$ in high temperature for the bended GNRs with $N_{L}=$ $N_{R}=6, W_{c}=5$, and $H_{c}=18$. Furthermore, the valley value is sensitive to the structural parameters and can be modulated by the interbend length $H_{c}$ as shown in Figure 5. For instance, the valley value rises to $28 \%$ for the system with $H_{c}=12$, and the contribution is always larger than that of the system with $H_{c}=18$ in the whole temperature range.

\section{Summary}

In summary, we have studied comparatively the thermal transport properties of FPMs and IPMs in the perfect GNR and bended GNR. Nonequilibrium Green's function approach is used to capture the atomistic phonon transport. We find that the thermal conductance of FPMs is slightly larger than that of IPMs in low high temperature and becomes more less than that of IPMs in the high temperature for perfect GNR. However, for the bended GNR, the alternative link of perfect zigzag and armchair GNRs leads to the phonon dispersion mismatch; the scattering occurs for all 


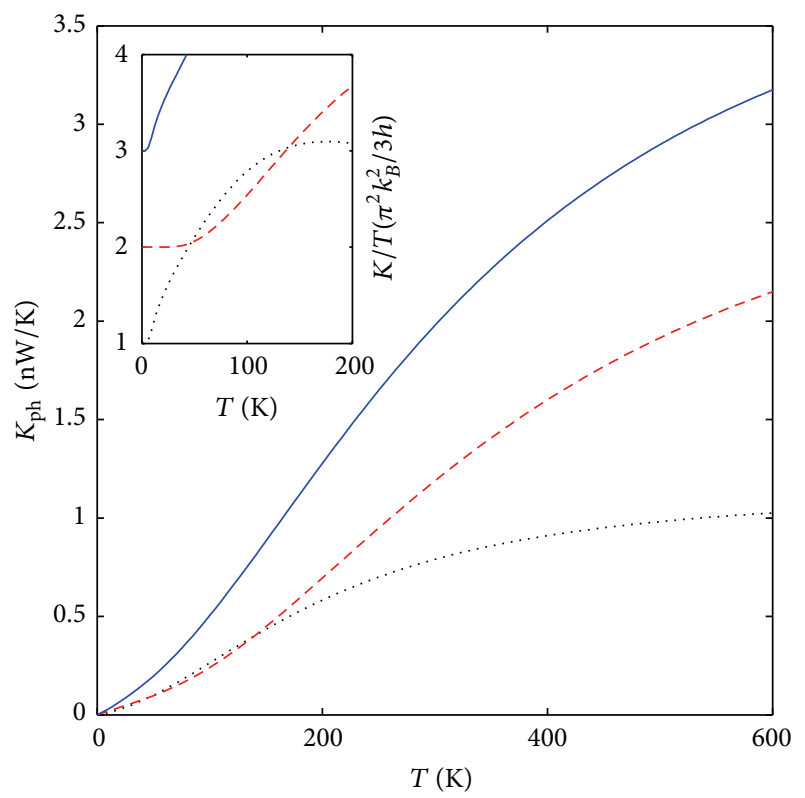

(a)

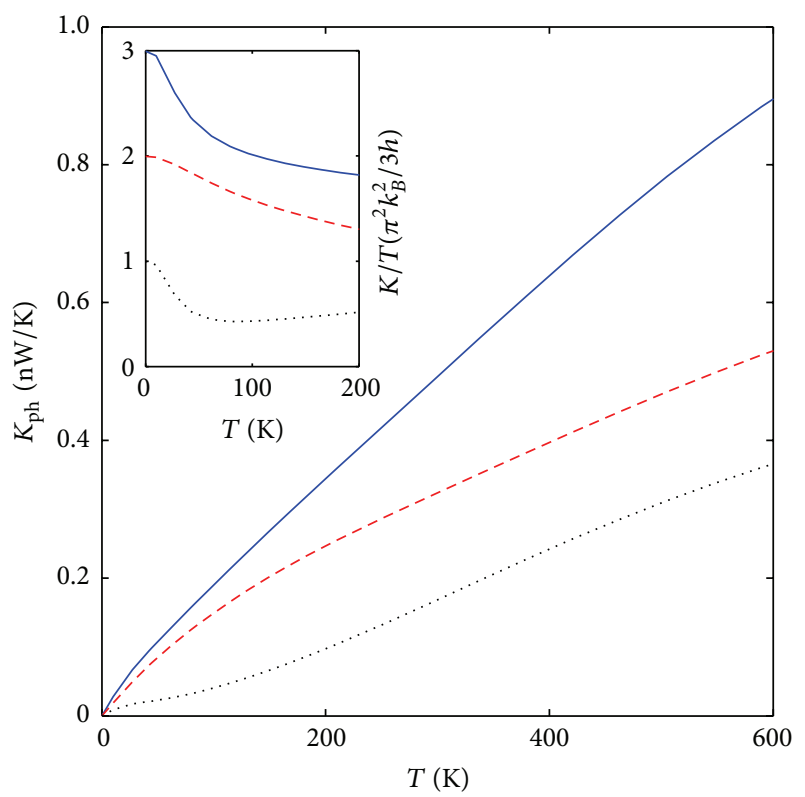

(b)

FIGURE 4: The thermal conductance and reduced thermal conductance (insets) as functions of the temperature for (a) the perfect 6ZGNR and (b) bended GNRs $N_{L}=N_{R}=6, H_{c}=18$, and $W_{c}=5$. Solid, dashed, and dotted curves correspond to the total, IPMs, and FPMs thermal conductance and their reduced thermal conductance (insets), respectively.

phonon modes. Both types of thermal conductance for FPMs and IPMs degrade more from perfect GNRs though they increase when temperature raises. But the effect of scattering for FPMs and IPMs is different and is sensitive to the structural parameters; it provides a way to modulate conveniently the transport of different phonon modes in the considered system. These results will be helpful for designing

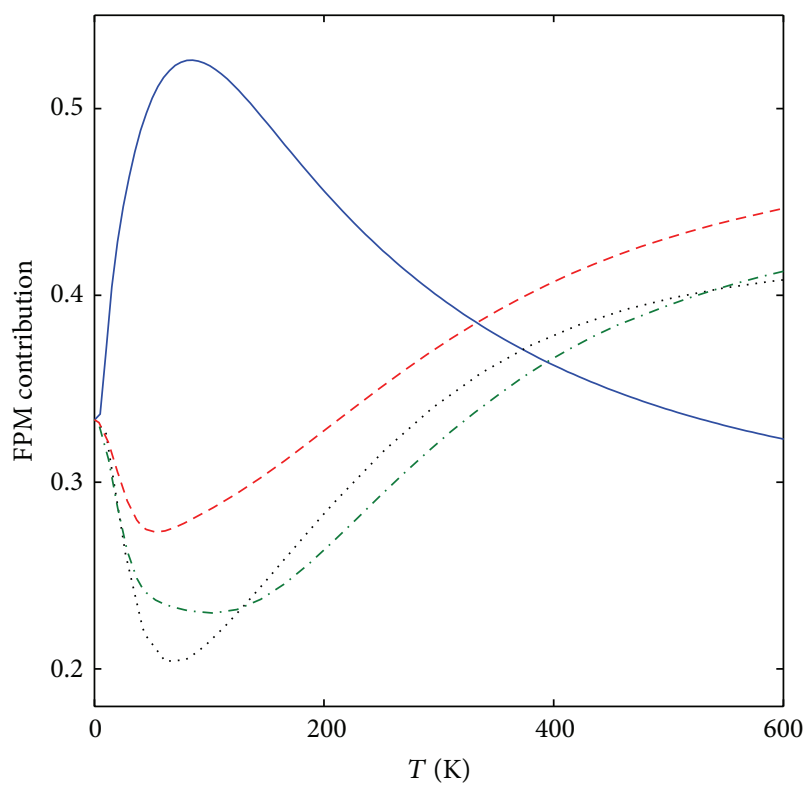

FIGURE 5: The contribution of the thermal conductance of FPMs to the total thermal conductance for the perfect 6-ZGNR (solid) and bended GNR with the fixed $N_{L}=N_{R}=6$ and $W_{c}=5$ and different $H_{c}=12$ (dashed), $H_{c}=14$ (dashed-dotted), and $H_{c}=18$ (dotted) as functions of the temperature, respectively.

high-performance thermal insulation or thermoelectric nanodevice based on graphene.

\section{Competing Interests}

The authors declare that there is no competing interests regarding the publication of this paper.

\section{Acknowledgments}

This work was supported by the National Natural Science Foundation of China (Grant nos. 61401153 and 11374094), by Hunan Provincial Natural Science Foundation of China (nos. 2015JJ2050 and 14JJ3126), and by the Open Research Fund of the Hunan Province Higher Education Key Laboratory of Modeling and Monitoring on the Near-Earth Electromagnetic Environments, Changsha University of Science and Technology (no. 20160105).

\section{References}

[1] Y.-M. Lin and P. Avouris, "Strong suppression of electrical noise in bilayer graphene nanodevices," Nano Letters, vol. 8, no. 8, pp. 2119-2125, 2008.

[2] A.-K. Geim, “Graphene: status and prospects," Science, vol. 324, no. 5934, pp. 1530-1534, 2009.

[3] C. Berger, Z. Song, X. Li et al., "Electronic confinement and coherence in patterned epitaxial graphene," Science, vol. 312, no. 5777, pp. 1191-1196, 2006.

[4] Y.-B. Zhang, Y.-W. Tan, H.-L. Stormer, and P. Kim, “Experimental observation of the quantum Hall effect and Berry's phase in graphene," Nature, vol. 438, no. 7065, pp. 201-204, 2005. 
[5] F. Bonaccorso, Z. Sun, T. Hasan, and A. C. Ferrari, "Graphene photonics and optoelectronics," Nature Photonics, vol. 4, no. 9, pp. 611-622, 2010.

[6] N. Tombros, C. Jozsa, M. Popinciuc, H. T. Jonkman, and B. J. Van Wees, "Electronic spin transport and spin precession in single graphene layers at room temperature," Nature, vol. 448, no. 7153, pp. 571-574, 2007.

[7] O.-V. Yazyev and S.-G. Louie, "Electronic transport in polycrystalline graphene," Nature Materials, vol. 9, no. 10, pp. 806-809, 2010.

[8] S. Ghosh, D. L. Nika, E. P. Pokatilov, and A. A. Balandin, "Heat conduction in graphene: experimental study and theoretical interpretation," New Journal of Physics, vol. 11, no. 9, Article ID 095012, 2009.

[9] A.-A. Balandin, S. Ghosh, W.-Z. Bao et al., "Superior thermal conductivity of single-layer graphene," Nano Letters, vol. 8, no. 3, pp. 902-907, 2008.

[10] J. H. Seol, I. Jo, A. L. Moore et al., "Two-dimensional phonon transport in supported graphene," Science, vol. 328, no. 5975, pp. 213-216, 2010.

[11] S. Ghosh, W.-Z. Bao, D.-L. Nika et al., "Dimensional crossover of thermal transport in few-layer graphene," Nature Materials, vol. 9, no. 7, pp. 555-558, 2010.

[12] D.-L. Nika, S. Ghosh, E.-P. Pokatilov, and A.-A. Balandin, "Lattice thermal conductivity of graphene flakes: comparison with bulk graphite," Applied Physics Letters, vol. 94, no. 20, Article ID 203103, 2009.

[13] D.-L. Nika, E.-P. Pokatilov, A.-S. Askerov, and A.-A. Balandin, "Phonon thermal conduction in graphene: role of Umklapp and edge roughness scattering," Physical Review B, vol. 79, no. 15, Article ID 155413, 2009.

[14] J. Hu, Y. Wang, A. Vallabhaneni, X. Ruan, and Y.-P. Chen, "Nonlinear thermal transport and negative differential thermal conductance in graphene nanoribbons," Applied Physics Letters, vol. 99, no. 11, Article ID 113101, 2011.

[15] H.-Q. Huang, Y. Xu, X.-L. Zou, J. Wu, and W.-H. Duan, “Tuning thermal conduction via extended defects in graphene," Physical Review B, vol. 87, no. 20, Article ID 205415, 2013.

[16] T. Ouyang, Y.-P. Chen, Y. Xie, G.-M. Stocks, and J.-X. Zhong, "Thermal conductance modulator based on folded graphene nanoribbons," Applied Physics Letters, vol. 99, no. 23, Article ID 233101, 2011.

[17] T. Ouyang, Y.-P. Chen, Y. Xie et al., "Ballistic thermal rectification in asymmetric three-terminal graphene nanojunctions," Physical Review B, vol. 82, no. 24, Article ID 245403, 2010.

[18] Z.-X. Xie, K.-M. Li, L.-M. Tang, C.-N. Pan, and K.-Q. Chen, "Nonlinear phonon transport and ballistic thermal rectification in asymmetric graphene-based three terminal junctions," Applied Physics Letters, vol. 100, no. 18, Article ID 183110, 2012.

[19] N. Yang, G. Zhang, and B.-W. Li, “Thermal rectification in asymmetric graphene ribbons," Applied Physics Letters, vol. 95, no. 3, Article ID 033107, 2009.

[20] F. Mazzamuto, V. Hung Nguyen, Y. Apertet et al., "Enhanced thermoelectric properties in graphene nanoribbons by resonant tunneling of electrons," Physical Review B, vol. 83, no. 23, Article ID 235426, 2011.

[21] H. Sevinçli and G. Cuniberti, "Enhanced thermoelectric figure of merit in edge-disordered zigzag graphene nanoribbons," Physical Review B, vol. 81, no. 11, Article ID 113401, 2010.
[22] W. Huang, J.-S. Wang, and G. Liang, "Theoretical study on thermoelectric properties of kinked graphene nanoribbons," Physical Review B, vol. 84, no. 4, Article ID 045410, 2011.

[23] L. Liang, C.-S. Eduardo, C.-G. Eduardo, and M. Vincent, "Enhanced thermoelectric figure of merit in assembled graphene nanoribbons," Physical Review B, vol. 86, Article ID 115438, 2012.

[24] Z.-X. Xie, L.-M. Tang, C.-N. Pan, K.-M. Li, K.-Q. Chen, and W. Duan, "Enhancement of thermoelectric properties in graphene nanoribbons modulated with stub structures," Applied Physics Letters, vol. 100, no. 7, Article ID 073105, 2012.

[25] T. Gunst, T. Markussen, A.-P. Jauho, and M. Brandbyge, "Thermoelectric properties of finite graphene antidot lattices," Physical Review B, vol. 84, no. 15, Article ID 155449, 2011.

[26] H. Karamitaheri, M. Pourfath, R. Faez, and H. Kosina, "Geometrical effects on the thermoelectric properties of ballistic graphene antidot lattices," Journal of Applied Physics, vol. 110, no. 5, Article ID 054506, 2011.

[27] C.-N. Pan, Z.-X. Xie, L.-M. Tang, and K.-Q. Chen, "Ballistic thermoelectric properties in graphene-nanoribbon-based heterojunctions," Applied Physics Letters, vol. 101, no. 10, Article ID 103115, 2012.

[28] M. Morooka, T. Yamamoto, and K. Watanabe, "Defect-induced circulating thermal current in graphene with nanosized width," Physical Review B, vol. 77, no. 3, Article ID 033412, 2008.

[29] Z.-X. Xie, K.-Q. Chen, and W.-H. Duan, “Thermal transport by phonons in zigzag graphene nanoribbons with structural defects," Journal of Physics: Condensed Matter, vol. 23, no. 31, Article ID 315302, 2011.

[30] N. Mingo, D. A. Stewart, D. A. Broido, and D. Srivastava, "Phonon transmission through defects in carbon nanotubes from first principles," Physical Review B, vol. 77, no. 3, Article ID 033418, 2008.

[31] X.-F. Peng, X.-J. Wang, Z.-Q. Gong, and K.-Q. Chen, "Ballistic thermal conductance in grapheme nanoribbon with doublecavity structure," Applied Physics Letters, vol. 99, no. 23, Article ID 233105, 2011.

[32] L. Lindsay, D.-A. Broido, and N. Mingo, "Flexural phonons and thermal transport in graphene," Physical Review B, vol. 82, no. 11, Article ID 115427, 2010.

[33] Y. Lu and J. Guo, "Thermal transport in grain boundary of graphene by non-equilibrium Greens function approach," Physical Review B, vol. 101, no. 4, Article ID 043112, 2012.

[34] Z. Wang, R. Xie, C. T. Bui et al., "Thermal transport in suspended and supported few-layer graphene," Nano Letters, vol. 11, no. 1, pp. 113-118, 2011.

[35] J.-S. Wang, J. Wang, and J. T. Lü, "Quantum thermal transport in nanostructures," European Physical Journal B, vol. 62, no. 4, pp. 381-404, 2008.

[36] S. Ghosh, I. Calizo, D. Teweldebrhan et al., "Extremely high thermal conductivity of graphene: prospects for thermal management applications in nanoelectronic circuits," Applied Physics Letters, vol. 92, Article ID 151911, 2008.

[37] D.-W. Brenner, O.-A. Shenderova, J.-A. Harrison, S. J. Stuart, B. $\mathrm{Ni}$, and S. B. Sinnott, "A second-generation reactive empirical bond order (REBO) potential energy expression for hydrocarbons," Journal of Physics: Condensed Matter, vol. 14, no. 4, pp. 783-802, 2002. 
[38] S.-H. Tan, L.-M. Tang, Z.-X. Xie, C.-N. Pan, and K.-Q. Chen, "Effect of pentagon-heptagon defect on thermal transport properties in graphene nanoribbons," Carbon, vol. 65, pp. 181-186, 2013.

[39] T. Yamamoto, K. Watanabe, and K. Mii, "Empirical-potential study of phonon transport in graphitic ribbons," Physical Review $B$, vol. 70, no. 24, Article ID 245402, 7 pages, 2004.

[40] Q.-F. Sun, P. Yang, and H. Guo, "Four-terminal thermal conductance of mesoscopic dielectric systems," Physical Review Letters, vol. 89, no. 17, Article ID 175901, 2002. 

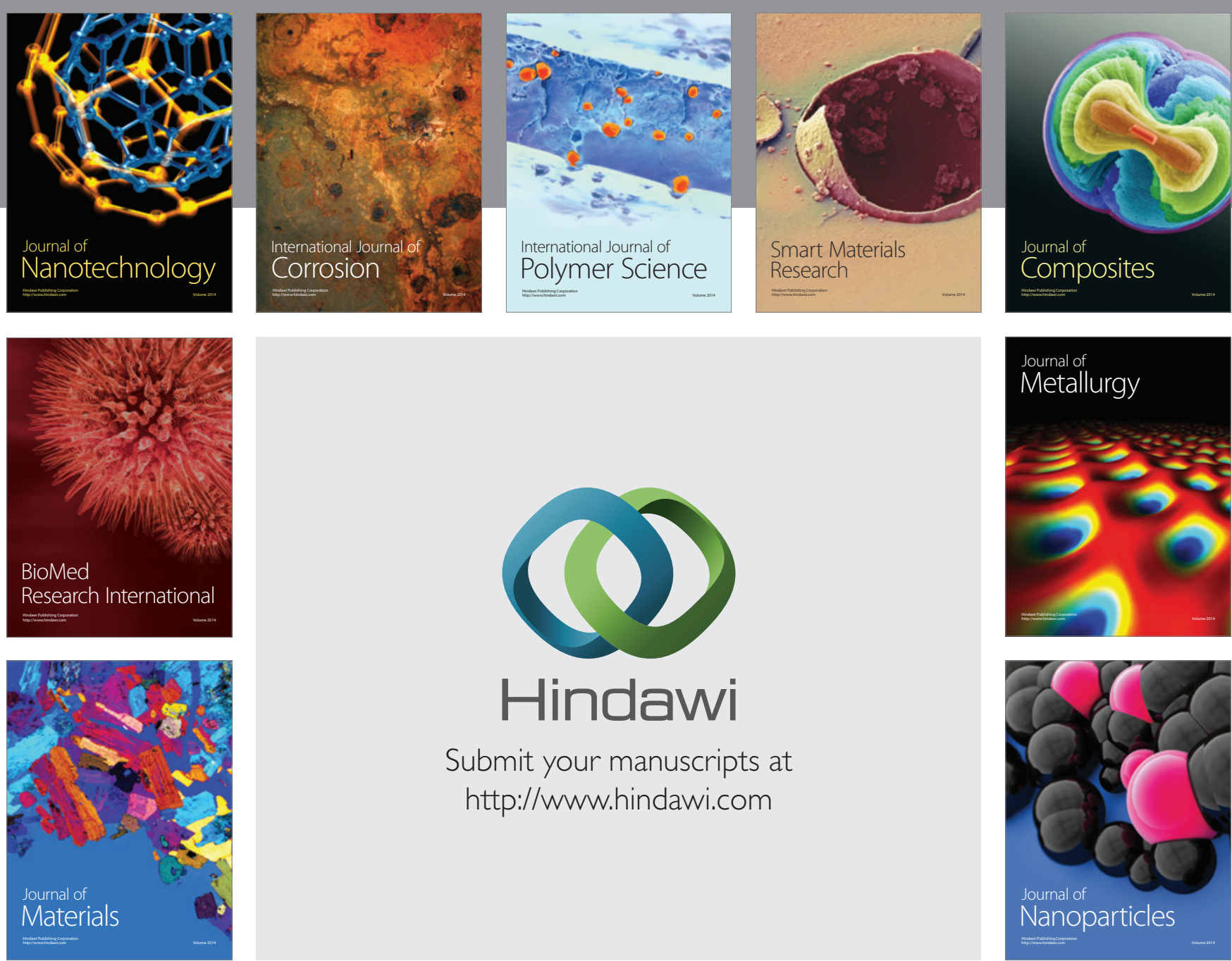

\section{Hindawi}

Submit your manuscripts at

http://www.hindawi.com

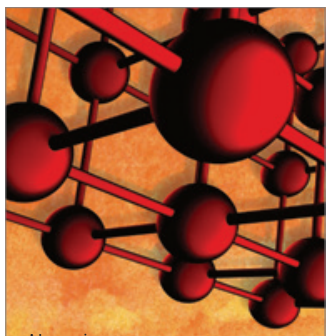

Materials Science and Engineering
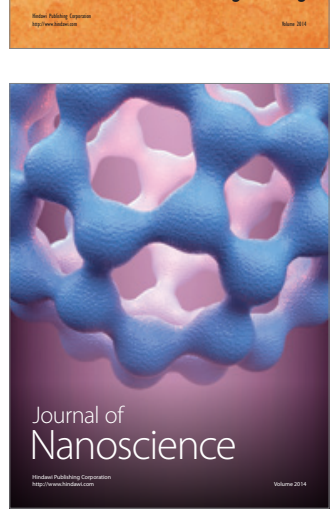
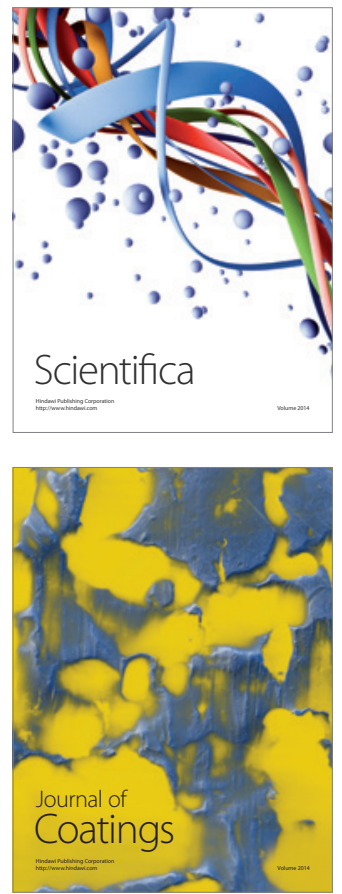
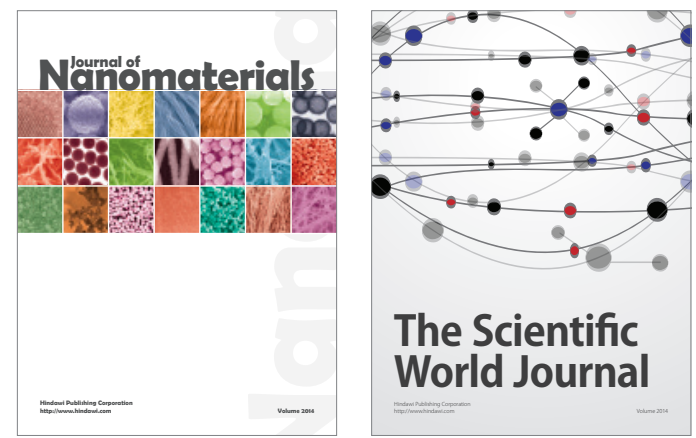

The Scientific World Journal
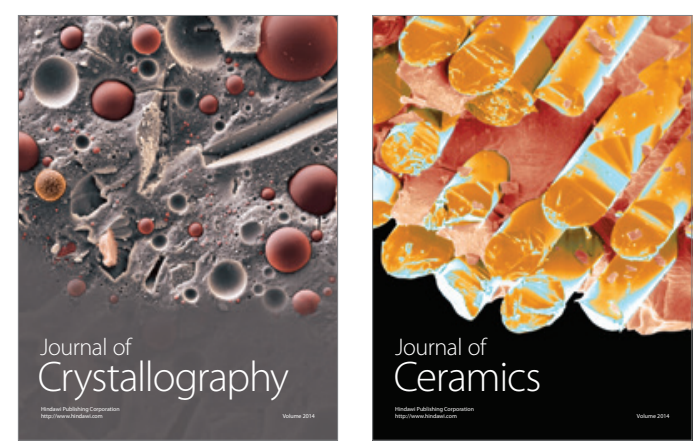
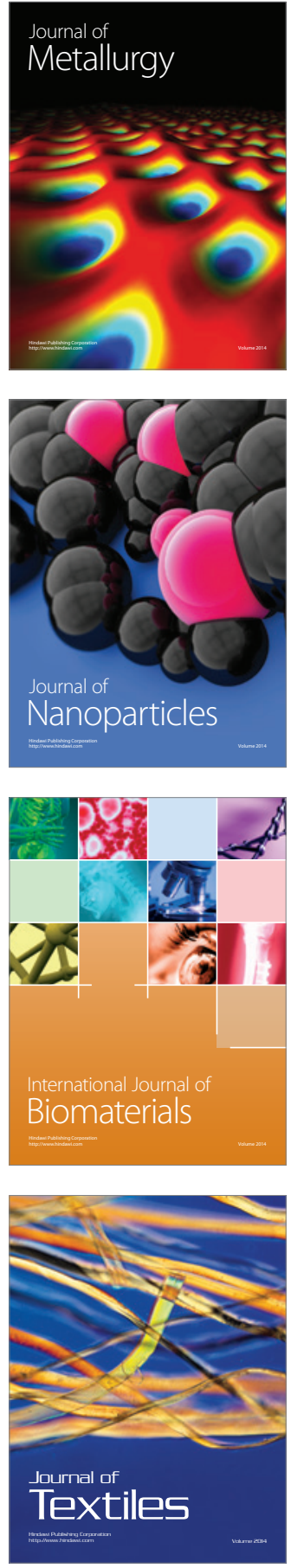